\title{
Intermédialités
}

Histoire et théorie des arts, des lettres et des techniques

Intermediality

History and Theory of the Arts, Literature and Technologies

\section{Théâtre d'ombres chez Sophie Calle : les mises en scène du moi et de l'absence}

\section{Magali Nachtergael}

Numéro 7, printemps 2006

Filer (Sophie Calle)

Shadowing (Sophie Calle)

URI : https://id.erudit.org/iderudit/1005521ar

DOI : https://doi.org/10.7202/1005521ar

Aller au sommaire du numéro

Éditeur(s)

Centre de recherche sur l'intermédialité

ISSN

1705-8546 (imprimé)

1920-3136 (numérique)

Découvrir la revue

Citer cet article

Nachtergael, M. (2006). Théâtre d'ombres chez Sophie Calle : les mises en scène du moi et de l'absence. Intermédialités / Intermediality, (7), 139-150.

https://doi.org/10.7202/1005521ar
Résumé de l'article

La mise en scène de l'absence, pour reprendre l'expression de Susan Sontag, est un élément essentiel de l'oeuvre de Sophie Calle. Tout en continuant à mêler habilement vérité et fiction, elle façonne son image d'artiste dans son grand théâtre d'ombre où la photographie montre autant qu'elle cache. Depuis ses filatures à Venise ou dans Paris, elle est passée maître dans l'art de la manipulation de ses personnages, qu'ils soient des monuments communistes démantelés, des jeunes filles disparues ou des tableaux volés. 


\title{
Théâtre d'ombres chez Sophie Calle: les mises en scène du moi et de l'absence
}

\author{
Magali Nachtergael
}

\begin{abstract}
l est troublant de constater à quel point l'œuvre de Sophie Calle se nourrit nécessairement de son paratexte, qu'il apparaisse fortuitement dans les journaux ou lors de ses revues parlées. À l'occasion des interviews qu'elle a données en 2003 pour son exposition M'as-tu vue au Centre Pompidou, un nouvel élément significatif est apparu, projetant le lecteur-spectateur attentif en de nouvelles conjectures: Sophie Calle lutterait en permanence contre l'oubli, ayant, dit-elle, une très mauvaise mémoire. Ses œuvres résulteraient donc de ses amnésies récurrentes: noter et prendre des photos, voilà qui supplée aux trous tant redoutés. Sa précédente exposition, Doubles-jeux, présentée au feu Centre national de la photographie en 1998, nous montrait déjà qu'elle avait accumulé un bagage de souvenirs conséquents. Il était donc temps de les cataloguer, ce qu'ont fait les éditions Actes Sud qui, en publiant les séries de Calle, assuraient en quelque sorte une pérennité salvatrice. Ces éditions ont marqué par ailleurs une nouvelle ère tant sur l'image artistique qu'éditoriale de son œuvre. L'effet « rétrospective » oblige toujours l'artiste à de nécessaires réajustements. De fait, une partie de l'œuvre de Sophie Calle est retournée dans les tiroirs ${ }^{1}$ pour lesquels elle semblait avoir été toujours conçue : Léviathan ${ }^{2}$ n'est plus son livre de chevet, la cabine téléphonique de New York qu'elle avait décorée sous l'injonction de Paul Auster a repris ses couleurs originelles et chacune des œuvres de Sophie Calle se réduit l'une après l'autre au format poche comme pour se faire plus commodes à ranger. La grande exposition du Centre Pompidou a fait un peu oublier celle du Centre national de la photographie. Laspect encore «bricolage » et l'intimité qui y régnaient ont cédé la place à un cénacle agrandi dont l’influence s'est
\end{abstract}

1. L'artiste range ses documents dans des boîtes où les photographies, lettres et carnets reposent en attendant d'être exploités ou non.

2. Paul Auster, Léviathan, trad. Christine Le Bœuf, Arles, Actes Sud, 1993 [1991]. 
étendue jusqu'aux couvertures des magazines. Après vingt années d'expositions de soi et des récits associés, l'œuvre apparaît enrichie de nouvelles thématiques qui ont trait à l'absence, à la mise en scène du vide et qui laisse la part belle à des voix venues remplacer les images. Dans cette perspective de renouvellement du regard critique sur l'œuvre de Sophie Calle, on interrogera cette esthétique de l'absence qui affecte le personnage même de Sophie Calle et qui «s'exfiltre» pour contaminer toutes les figures que le lecteur rencontre dans les textes. Enfin, nous verrons comment, par l'usage de la photographie, l'écrit se trouve traversé, comme brouillé par les scories d'une tradition poétique inattendue dans une œuvre si contemporaine qui réactive avec subtilité les liens entre image et texte.

\section{ÉVACUER, EFFACER}

À la suite des Histoires vraies 3 publiées en 1994 dans leur mouture originale, l'expérience Doubles-jeux, placée de façon manifeste sous le signe romanesque de Paul Auster, a donné l'occasion de traiter à fond les questions de fiction et de vérité. Le film No Sex Last Night (1992) avait par ailleurs marqué le point culminant de l'intrication entre vie réelle et vie artistique pour Sophie Calle. Une fois ces thèmes exploités par l'artiste jusqu'à leurs dernières limites, il était temps d'en venir à l'autre élément qui circule dans toute l'œuvre même de Sophie Calle, à savoir sa mise en scène de l'absence, perceptible en filigrane depuis longtemps, mais souvent considéré comme un dispositif de circonstance, subordonné aux thèmes abordés dans les récits. Christine Angot ne s'y trompe pas quand elle interroge une Sophie Calle fictive:

Qu'est ce qui vous distingue des autres? Ça, que je n'ai pas la chose, et que je la veux, je ne comprends pas pourquoi il ne me la donne pas ce con. Pourquoi ne me la donnent-ils pas ces cons? J'ai tout le reste, maison, talent, succès. C'est si simple pour lui, il suffit qu'il couche avec moi, et justement, alors que je me suis mariée avec lui, et que je l'aime, et qu'il m'aime peut-être, je suis aimable, je suis belle, intelligente, talentueuse, il ne me la donne pas, c'est ça la sexualité féminine, le continent noir, le gouffre, le monstre. Le manque. L'absence4.

Un des clichés des Histoires vraies montre le corps d'un homme, sans tête. Son sexe a disparu entre les jambes, ne laissant apparaître qu'un vague androgyne. Son titre en est Lamnésie et Sophie Calle explique dans son habituel commentaire qu'elle ne parvient jamais à se souvenir du sexe des hommes ou de

3. Sophie Calle, Histoires vraies [1994], dans Histoires vraies + dix, Arles, Actes Sud, 2002.

4. Christine Angot, «No sex», dans Beaux Arts Magazine, n² 234, novembre 2003, p. $80-84$. 
la couleur de leurs yeux. Cette image aux marges de l'oubli et de la fiction peut former le pivot entre Doubles-jeux ${ }^{5}$ et le coffret Labsence ${ }^{6}$ : ce dernier coffret en forme de triptyque a montré au public français un volet en apparence secondaire de l'œuvre de Sophie Calle, ou à tout le moins, négligé peut-être en raison de son «informité ». Il retient néanmoins deux caractéristiques fondamentales, la mise en scène de l'absence et la voix de l'autre.

En effet, depuis le début de sa carrière, Sophie Calle met en scène des petits ou des grands vides. À ce titre, la photo et le texte arborent une valeur référentielle d'autant plus forte: si l'on considère que l'absence est toujours absence de quelque chose, de quelqu'un ou finalement, de soi-même, l'acte autobiographique n'est pas si incompatible avec cette représentation obsessionnelle de l'absent. En effet, l'autobiographie, selon Jean-Bertrand Pontalis «[...] apparaît souvent comme une nécrologie anticipée, comme le geste ultime d'appropriation de soi et par là peut-être comme un moyen de discréditer ce que les survivants penseront et diront de vous, de conjurer le risque qu'ils n'en pensent rien ${ }^{7}$.

Après avoir édifié son autobiographie sous plusieurs formes, Sophie Calle ne se rapproche-t-elle pas des disparus, des absents pour mieux se dissoudre ${ }^{8}$ dans son rôle, tel le funambule de Genet toujours en équilibre, et disparaître à son tour complètement? Face aux aveugles, les Tirésias modernes, elle s'effaçait déjà pour leur laisser la parole; dans Suite vénitienne, elle disparaît, selon les mots de Jean Baudrillard dans «la trace de l'autre9". Dans Fantômes, Disparitions, Souvenirs de Berlin-Est, le message paraît clair : il n’est question que de ce qui «a

5. Sophie Calle, Doubles-jeux, Arles, Actes Sud, 1998. Le coffret de sept livres reprend des œuvres citées dans le Leviathan de Paul Auster ou exécute des idées attribuées à l'alter ego romanesque de Sophie Calle, Maria Turner. L'exposition et la publication associée fonctionnaient comme une rétrospective majeure de la production de Sophie Calle.

6. Sophie Calle, L'absence (Souvenirs de Berlin-Est, Fantômes, Disparitions), Arles, Actes Sud, 2000, 3 vol. Ce coffret de trois livres compile des œuvres réalisées entre 1991 et 1999 en liaison avec la disparition d'œuvres, de personnes et les traces qu'ils laissent physiquement ou dans les mémoires.

7. Jean-Bertrand Pontalis, «Premiers mots, derniers mots», dans Philippe Lejeune, Michel Neyraut et al., L'autobiographie. VI es Rencontres psychanalytiques d'Aix-en Provence, Paris, Société d'éditions Les Belles Lettres, coll. «Confluents psychanalytiques ", 1988, p. 51.

8. Jean Genet, Le condamné à mort et autres poèmes, suivi de Le funambule, Paris, Éditions Gallimard, Collection «Poésie», 1999.

9. Jean Baudrillard, «Please follow me», dans Sophie Calle, Suite vénitienne, Paris, Éditions de L'Étoile, coll. «Écrit sur l'image», 1983, p. 81. 
été là » et non pas d'elle. Pourtant, c'est dans la mise en scène et, comme à son habitude, dans la manipulation de ces voix qu'elle entérine le processus artistique dans lequel elle s'est toujours confondue.

On pourrait relier ces dernières œuvres du coffret L'absence, plutôt avec une idée du théâtre antique qui se jouerait dans les musées et les livres, qu'avec un panorama de l'art contemporain par rapport auquel elle semble décalée. On perçoit en effet des procédés tragiques propres à la poétique classique, tels que l'évocation des morts (ou plutôt des « ombres») mais aussi l'ekphrasis, description de l'œuvre absente qui ravive la tradition de l'ut pictura poesis, d'après laquelle «Il en va de même pour la peinture et la poésie». Sophie Calle, dans son utilisation mixte du texte et de l'image, opère une synthèse moderne de la célèbre formule d'Horace pour produire une fabrique nouvelle du personnage: ce dernier acquiert alors une existence autant dans l'image que dans le texte, et la figure de l'ekphrasis participe à la dématérialisation de ces personce.

\section{LES PERSONNAGES DE SOPHIE CALLE}

Mais de quelle étoffe sont faits les personnages évoqués chez Sophie Calle? Il y a en effet beaucoup de figures qui traversent les images, se faufilent dans les récits. Ces personnages glissent comme des ombres qui ne font que passer, ou encore comme des marionnettes que Sophie Calle, en tant que grand metteur en scène, manipule de loin en proche. En décomposant cette scénographie, outre les décors en extérieurs, Paris ou Venise, s’imposent les termes d'imago, persona, fictio qui forment les ingrédients d'une «évocation» de ces ombres. Pour cela, revenons un instant à un des matériaux qui compose l'œuvre: l'image photographique. Les récits de Sophie Calle appellent en effet un processus de lecture où le médium qui y est inséré, en l'occurrence la photographie, joue un rôle significatif dans la réception même du dispositif. Roland Barthes peut nous guider un temps sur le chemin qui mène à la définition des signes particuliers de l'image. Dans La chambre claire, il compare, comme beaucoup d'autres, la photographie à la scène: «la Photo est comme un théâtre primitif, comme un Tableau vivant, la figuration de la face immobile et fardée sous laquelle nous voyons nos morts $^{10}$. » Est-il besoin de rappeler que l'imago était le masque de cire que l'on modelait - fingere qui a donné le mot «fiction» - sur les morts et que l'on

10. Roland Barthes, La chambre claire. Note sur la photographie, Paris, Éditions des Cahiers du cinéma, Éditions Gallimard, Éditions du Seuil, 1980, p. 56. 
accrochait aux murs du foyer ${ }^{11}$. Arborés par les acteurs, ces masques figuraient leur persona ou «rôle», terme qui a donné le «personnage», un personnage presque directement moulé sur le visage du mort. La fiction semble parachever de façon redondante cette transmutation du personnage en spectre photographique, «imago photographique ${ }^{12}$ » nous disent encore Roland Barthes ou Susan Sontag. Sophie Calle aussi, sous l'effet de la photographie, se fige en une persona dont le masque montre autant qu'il cache.

Elle utilise en effet dans ses travaux autobiographiques sa propre identité et son image comme un écran de fumée - ou de projection - derrière lequel elle peut disparaître, se dissoudre dans la fiction, et atteindre quelque chose qui a trait à sa mort dans sa propre mise en scène. Comme elle se plaît à dire dans le questionnaire psychologique de Damien Hirst: «dans mes rêves, on m’enterre, mes funérailles sont bouleversantes, je ressuscite, je ne suis pas morte ${ }^{13} »$, comme si la mort n'était qu'un simulacre et que les mises en scène préliminaires à ses œuvres n’étaient que des répétitions générales pour la dernière représentation, horizon de chaque autobiographie. En 1999, à la même époque que Doubles-jeux, Yann Toma avait poussé le principe à son extrémité en proposant à qui le souhaitait de prendre un cliché de sa propre mort. Sous le titre Crimes sur commande (19982005), des meurtres, suicides, morts violentes étaient mis en images en contrepartie d'une somme modique qui entérinait l'acte d'achat de l'œuvre.

Sur cette scène de théâtre photographique, le personnage de Sophie Calle, dont les contours sont toujours fuyants, joue à cache-cache avec les ombres: le titre anglais de La filature, The Shadow, figure bien cette disparition spectrale où le suiveur devient l'ombre d'un autre. «Shadowing», en français, ce serait l'art de filer à l'anglaise, ou disparaître en douce, mais c'est aussi le préliminaire à la broderie, un motif récurrent au propre et au figuré chez Sophie Calle. On pense aux draps de la grand-mère dans les Histoires vraies et bien sûr à Douleur exquise dont le texte est brodé sur les grands pans de tissu noir, ainsi qu'aux multiples panoplies de vêtements qui habillent ses personnages. Une filature, c'est aussi une manufacture de fils, une longue broderie, un tissu que l'on coud, tel le rhapsode qui

11. La série restée discrète, Statues ennemies (2003), montre cette image blessée des visages en pierre qui apparaissait déjà dans L'homme au carnet lors d'un passage de Sophie Calle au Père Lachaise.

12. Roland Barthes, La chambre claire, p. 21 et Susan Sontag, Sur la photographie, trad. Philippe Blanchard, Paris, Éditions Christian Bourgois, 2003 [1977].

13. Sophie Calle, «Évaluation psychologique sur une idée de Damien Hirst», dans Sophie Calle, M'as-tu vue, catalogue de l'exposition, Christine Macel (éd.), Paris, Éditions du Centre Pompidou, Éditions Xavier Barral, 2003, non paginé. 
«ajustait» ses chants entre eux, comme des pièces de vêtements. Aujourd'hui, Sophie Calle arrange encore des histoires, mais avec la voix des autres.

La lecture de l'œuvre de Sophie Calle a été modifiée en grande partie depuis que son éditeur a permis une vue globale de ses travaux depuis vingt ans. Cette absence qui jouait auparavant la basse continue, maintenant, par un effet de vases communicants, laisse place à la voix de Sophie Calle qui susurre en permanence: «ce n'est pas moi, mais c'est toujours moi ». Sa voix, venue de derrière le masque, paraît irréelle, hors champ, hors scène: le même effet de sourdine devait être ressenti par les spectateurs des théâtres antiques. Au Confessionnal, une installation datée de 1983, elle lisait le texte de sa filature à Venise au visiteur assis dans la boîte noire, comme une ombre invisible et un peu inquiétante.

Il est aisé par conséquent d'accuser Sophie Calle, comme une criminelle, d'être manipulatrice, ce qu'Hervé Guibert n'a pas hésité à faire: et quoi de plus normal dans cette posture que de tendre les fils de ses marionnettes à distance? Les photos sont telles les marionnettes: reposez-les au sol, leurs fils s'emmêlent et deviennent incompréhensibles. Les histoires de Sophie Calle n'ont rien d'intelligible si elle ne les raconte pas et si elle ne brode pas un peu, si elle ne défait pas les fils pour animer sa petite histoire, il ne se passe rien, ou alors, pas grand-chose.

Les personnages évoluent en ombres chinoises, ils n’apparaissent jamais vraiment, désignés par des prénoms ou des initiales, un amas d'objets dans une chambre d'hôtel, une accumulation de pronoms: un «il» qui répond le plus souvent au « je », selon les formules récurrentes: «il était... il avait... je lui ai demandé », etc. Ces passants sont en général des absents qui ont laissé quelques traces. Ici, une jeune femme en boa et son appartement dévasté (donc, plus rien, si ce n'est de l'informe) ou encore des fantômes de tableaux, des socles de monuments ou des poteaux qui relient des fils imaginaires autour de Jérusalem... Encore des fils, qui ceux-là aussi parfois disparaissent, pour n'être que des supports à la matrice narrative (autobiographique ou non) qui commence généralement par des formules stéréotypées: "Je fus invitée... je me suis rendue pour la première fois à Berlin... J'ai demandé à des aveugles de naissance », qui expliquent les coulisses des images.

Ces coulisses de la photographie, Sophie Calle nous les donne dans ses textes, mais pas à n’importe quel moment. Il s'agit généralement d'un incipit plus ou moins bref qui expose la machination qui va nouer le drame. Le strip-tease ${ }^{14} \mathrm{en}$ est un exemple flagrant, car son schéma narratif est presque idéal : paradis perdu, spectacle routinier, drame et fin tragique. Elle semble utiliser volontiers un deus

14. Sophie Calle, Les panoplies. Doubles-jeux (Livre III), Arles, Actes Sud, 1998, p. 19. 
ex machina qui dénoue le drame qu'elle a elle-même créé, tantôt un «étranger providentiel » ou un «coup de dés» qui viennent la sortir d'une impasse imaginaire. Le spectacle qu'elle donne, l'image d'elle-même qu'elle crée et les personnages qui passent et gravitent dans les clichés se trouvent en fait parcellés, éclatés, reconstitués dans les textes (étymologiquement, le tissu), reliés par le contexte et parfois même «cousus de fil blanc».

\section{LA MISE EN SCÈne de L’ABSENCE: đuVRES FANTÔMES, IMAges fantôMES}

Parmi les mécanismes de mise en absence, l'affirmation permanente de la figure de Sophie Calle fait de sa disparition un événement, spécialement lorsque sa voix se tait au profit de celle des autres, comme si Sophie Calle abdiquait devant l'autre et, enfin, cessait de se raconter pour céder la place à d'autres récits. Néanmoins, même dans ce cas, l'effacement reste relatif. Les paroles émanent en effet de personnes anonymes ou aveugles, fantômes d'elles-mêmes, dont la fonction est de décrire un objet qui a été ôté de leur vue. Le lecteur n’a plus alors comme référent stable que l'identité de l'auteur, attestée par l'incipit à la première personne du singulier. L'oscillation entre l'absence de l'auteur et sa présence implicite dans la mise en scène des récits sous-tend l'idée évoquée par Christine Macel d'une «mort de l'auteur ${ }^{15}$ » contre laquelle Sophie Calle lutterait. En construisant une œuvre monument qui a pour socle la disparition, elle élude continuellement la sienne. Dans ce climat où Sophie Calle est réputée omniprésente, son effacement, son absence devient plutôt un non-événement puisque l'œuvre ne se constitue plus que sur du vide, comme « des escaliers qui ne mènent à rien ${ }^{16}$ », dit Olivier Rolin, où les fantômes prennent place pour tenir lieu d'œuvre d'art.

Le coffret Absence est divisé en trois volumes, Fantômes, Disparitions et Souvenirs de Berlin-Est. Le contraste avec le précédent coffret Doubles-jeux, dans la présentation, la sobriété et le principe artistique est assez saisissant: de la Sophie Calle clinquante, travestie en Brigitte Bardot, on passe à un long chapelet de monuments communistes, aux allures désuètes, à l'esthétique soviétique lourde, mis en parallèle avec les descriptions qu'en font les habitants du quartier. C'est ce principe de reconstitution policière, de portrait in absentia que l'artiste présente, en remplacement des œuvres disparues dans Fantômes et Disparitions. Sophie Calle y est plus discrète, plus effacée. «Je » y est plus que jamais un autre, projeté dans une vraie altérité (les gardiens du musée) : multiple, polyphonique,

15. Christine Macel, «La question de l'auteur dans l'œuvre de Sophie Calle. Unfinished», dans M'as-tu vue, p. 18.

16. Olivier Rolin, «La tigresse de papier », dans M'as-tu vue, p. 138. 
polymorphe, finalement si informe que ce «je» disparaît aussi avec les œuvres volées derrière l'indéfinition de ses possibles références. Par exemple, dans Fantômes: "Je crois qu'on voit la partie supérieure de son corps [...] Je vois surtout de la couleur [...] Je vois des taches de couleur [...] Je me souviens de deux formes $[\ldots]^{17}$ », chaque témoignage étant séparé par un petit signe typographique signalant le changement d'énonciateur. Le pronom ne renvoie en définitive à rien, les œuvres ont disparu. Le dépouillement marque l'épuisement du lieu par les moindres détails et souvenirs disponibles, comme pour une archéologie du quotidien, selon l'expression consacrée.

Sophie Calle se plie en fait dans ces séries à une variation de l'exercice de l'ekphrasis, que Philostrate avait mis au point dans ses Eikones (Images) ${ }^{18}$. Il s'agit de réaliser avec art la description d'une galerie de tableaux, faisant preuve d'autant d'habilité, voire plus même que les peintres : ici, les descriptions se font à partir de souvenirs, réponse tactique pour les trous de mémoires dont semble souffrir l'artiste. Dans l'incipit de Disparitions, l'auteur parle de «mise en scène involontaire de l'absence ${ }^{19}$ » : l'adjectif «involontaire» trahit ici la récupération d'une situation qui n'a pas été provoquée par l'artiste et qui, en plus, est «écrite » par d'autres (de la même façon que les photos sont prises par d'autres).

Le premier «fantôme » - nom du cartel qui remplace une œuvre déplacée ou prêtée - date de $1989^{2 \circ}$. Elle explique, comme à son habitude, sa démarche en incipit de Fantômes :

En juin 1989, je fus invitée à participer à une exposition au Musée d’Art moderne de la Ville de Paris. Le tableau de Bonnard, Nu dans le bain, ayant été temporairement prêté, devant son emplacement vide, j’ai demandé aux conservateurs, aux gardiens et à d'autres permanents du musée de me le décrire et de me le dessiner. J'ai remplacé le tableau manquant par ces souvenirs ${ }^{21}$.

Ce travail a donc lieu trois ans avant No Sex Last Night (1992) et en pleines «autobiographies» alors qu'il ne sera rendu public que plus tard, donnant l'impression que cette démarche est récente quand elle est diffusée par les éditions Actes Sud en 2000. Last seen, titre original de Disparitions, a été réalisé en

17. Sophie Calle, Fantômes, p. 8 à 12.

18. Philostrate, Galerie de tableaux, Paris, Éditions Les Belles Lettres, coll. «La roue à livres », 1991.

19. Sophie Calle, Fantômes, p. 12.

20. Ce musée est celui-là même où elle avait fait sa première exposition collective en 1981 et y avait présenté Les dormeurs.

21. Sophie Calle, Fantômes, p. 5. 
1991: depuis cette année, Sophie Calle a vécu plusieurs deuils, l'édition de 2000 s'ouvre en effet sur un triple hommage, «Trois morts, trois absences ». Ce coffret, Absence, placé sous l'égide d'un testament (celui d'Isabella Stewart Gardner) laisse aussi planer plus que jamais le spectre d'Hervé Guibert et de «son image fantôme». Elle y fait également allusion à son dernier projet, Douleur exquise et au suicide de son ami, alors qu'elle était à quelques kilomètres de lui : «Je n'ai rien vu » répète-t-elle deux fois ${ }^{22}$ et l'on croit entendre ici le vague écho du «tu n’as rien vu à Hiroshima» répété par «Lui» dans Hiroshima mon amour (Alain Resnais, 1959). Dans ce triptyque, les voix qui s'élèvent pour dire l'absent s'apparentent à un coryphée venu chanter autour des places vides. Cette mise à distance rétablit en quelque sorte la fonction «régulatrice» et distanciée de la tragédie qui s'est jouée en coulisses, en hors champ.

Depuis 1989, les œuvres profitent d'une dynamique paradoxalement fondée sur l'absence : cette production modifie par conséquent la perception de la figure de Sophie Calle au sein de son œuvre. L'identité de Sophie Calle peut être considérée comme une matière première qui est manipulable et malléable au même titre que les témoignages qu'elle recueille. Son corps est en passe de devenir - mais différemment de celui d'Orlan qui filme ses opérations de chirurgie esthétique - une œuvre d'art: ces gardiens de musée qu'elle interroge ne sont-ils pas les bonzes bienveillants qui devront plus tard veiller sur ses restes? Car cette mise en scène de l'absence de laquelle Sophie Calle semble s'éclipser n'est toujours qu'une représentation d'elle dans sa fonction d'artiste, une artiste qui expose ses œuvres comme un prolongement d'elle-même et comme les traces de sa présence.

On peut ainsi parler d'un retour de l'artiste aux sources photographiques où la «vue» et les visions soulèvent une problématique essentielle de l'image. Les aveugles avaient marqué une période où l'artiste voulait s'effacer, puisqu'on lui reprochait de trop se mettre en avant. L'expérience témoigne en outre du véritable intérêt de Calle pour la vue, ce qui n’a pas manqué de la rapprocher de Guibert qui travaillait alors à l'Institut National des Jeunes Aveugles de Paris. Cependant, comme la chronologie des œuvres n'est pas respectée en raison de l'effet éditorial, une fois de plus, les pistes sont brouillées. La série Last seen montre bien que cette inquiétude relative à l'absence s'est forgée petit à petit pour prendre un place à part entière au fil des ans. «Last seen », c'est la formule qui orne les avis de recherche aux États-Unis («vu la dernière fois le...»), là où Sophie Calle avait elle-même disparu pendant quelques années. C'est la même inspiration qui lui a donné le titre de sa dernière exposition. La formule «M'avez-

22. Sophie Calle, Disparitions, p. 7. 
vous vue? » sur une affiche, accompagnée d'une photo, avait frappé l'artiste par son adresse directe. De plus, fin 2003, Sophie Calle envisageait un projet sur des personnes disparues, leur donnant ainsi une place officielle dans ses œuvres ${ }^{23}$. Ces figures, dont Bénédicte Vincens aurait été la première à entrer en scène, deviennent de véritables personnages absents, des coquilles vides qui peuvent servir de personce à Sophie Calle. Ils la font sortir encore plus d'elle-même et lui permettent de s'éloigner résolument de cette image égo-centrée forgée dans Doubles-jeux, bien qu'elle ne puisse jamais se défaire complètement de ce nom qui fonctionne entre vérité et fiction comme une «charnière identitaire ». Ainsi, l'image de Sophie Calle se trouve elle-même fondée sur un vide, un interstice où le doute et l'imaginaire peuvent se déployer librement.

POUR EN FINIR

En somme, il semblerait que toutes ses œuvres ne soient qu'une variation sur le thème inaugural rencontré dans le cimetière californien où elle a pris ses premières photos, comme le veut sa mythologie autobiographique ${ }^{24}$. Yve-Alain Bois note à cet égard: «La seule série venue du silence est Les tombes: serait-ce parce que les images diraient la vérité photographique, parce qu'il n'y a rien à dire de trivial sur la mort ${ }^{25}$ ? » Cette série a eu longtemps valeur d'hapax, mais est désormais complétée par Statues ennemies (2003), une de ses dernières réalisations sans textes.

Bien que ce ne soit pas l'endroit d'étudier plus précisément l'écriture blanche de Calle, on peut néanmoins soulever in extremis la question de la parole face à l'image: comment accorder du texte à de l'image et pourquoi un sentiment d'échec pour Les tombes ou pour Unfinished (2003-2005)? Qu'y a-t-il dans la représentation de l'absence qui ouvre ou ferme l'écriture? Pourquoi l'absence d'image porte parfois le texte et inversement, pourquoi l'image peut aussi bien mettre en scène ce sentiment d'absence? Il y a dans le « récit-photo » un rapport à l'absence et à l'écriture qui dispense une certaine forme de poésie. Pour faire

23. Le hasard a voulu que ce soit avec Florence Aubenas, journaliste française retenue en otage en 2004 pendant 157 jours en Irak, qu'elle ait commencé ce travail.

24. Cette série reste relativement confidentielle et l'artiste ne la considère pas comme une «œuvre réussie». Sophie Calle, Pierre tombale, Catalogue d'exposition présenté par Louis Vincent Thomas, Palerme, Éditions Novecento et Centre culturel de Palerme, 1992.

25. Yve-Alain Bois, «Contre l'image », dans Sophie Calle, À suivre..., Paris, Éditions du Musée d'Art moderne de la Ville de Paris, 1991, p. 5. 
un lien direct avec la série Les tombes, le recueil de poème de Jacques Roubaud, Quelque chose noir, un titre tiré du journal d'Alix-Cléo Roubaud, permet d'évoquer un peu la nature poétique de cette série silencieuse. Au sujet d'une photographie de la tombe de Wittgenstein, Jacques Roubaud dit qu'elle était «une tombe d'une seule pierre, longue, plate, sans couleur, dans l'herbe, sans ornements, une modestie féroce » et qu'il l'avait mise au mur comme si elle avait été: «La tombe de la photographie, prélevée de la tombe ${ }^{26}$ ». Il plane un mystère identique dans le phrasé poétique et ces images de pierres tombales. Chez Calle, les phrases sont également tendues, sobres et simples, elles s'en tiennent au minimum, donnant ainsi une impression de confession. Et ces phrases courtes et ramassées sont comme retenues par une émotion qui les empêcherait de s'étendre, qui les recroqueville sur elles-mêmes pour n'en faire qu'un noyau narratif insécable. Cette écriture «blanche » s'approche d'une autre poétique que Barthes avait décelée entre la photographie et le haïku, une poésie qui frappe par petites touches le moment. Cette pratique qui pose sur les détails et les choses un nouveau regard, que les auteurs du Nouveau Roman avaient adoptée pour son épure et son objectivité, Sophie Calle, à travers son usage du rapport d'événements, pourrait bien en être la descendante directe, sous l'influence croisée de Georges Perec (on pense à sa Tentative d'épuisement d'un lieu parisien, 1975²7).

En guise de conclusion, la Suite vénitienne et quelques mots de Jean Baudrillard serviront à jeter un pont entre deux époques d'une carrière artistique toute en nuances, fruit de progressions, d'ajouts et de manques. Cette boucle met en lumière les liens qui unissent le coffret Absence, Une jeune femme disparaît (2005) et Les journées passées sous le signe du C (et spécialement la journée «Calle au Cimetière ${ }^{28}$ ») à ces images de sépultures qui rappellent insidieusement la mort - physique - de l'auteur. Bénédicte Vincens, admiratrice de l'artiste, voulait vivre comme elle. La nuit où son appartement a pris feu, elle s'est enfuie sans laisser de traces. Elle travaillait au Centre Pompidou comme gardienne et en juste (?) retour des choses, Sophie Calle décida de prendre sa place pendant son exposition M'as-tu vue. L'étrange jeu de substitution au musée affirme la survivance de Sophie Calle, sa permanence, ce qu'elle faisait déjà face à son caveau, devant lequel elle s'est fait photographier plusieurs années de suite. Ce

26. Jacques Roubaud, Quelque chose noir, Paris, Éditions Gallimard, coll. «Poésie», 1986 , p. 46

27. Georges Perec, Tentative d'épuisement d'un lieu parisien, Paris, Éditions Christian Bourgois, 1975 .

28. Sophie Calle, De l'obéissance. Doubles-jeux (Livre I), Arles, Actes Sud, 1998, p. 46. 
côté roman noir, un peu macabre, nous révèle aussi que tant qu'elle est là, elle peut encore défier l'absent, jouer à sa propre disparition, mettre en scène celle des autres. Jean Baudrillard, dans la préface de Suite vénitienne, expliquait déjà son «cérémonial» orphique et la «volupté de l'éminence grise: l'art de faire disparaître l'autre. » Le crime se double d'un paradoxal désir de dissolution dans la trace du suivi: Baudrillard module son commentaire lorsqu'il suggère que «le réseau de l'autre est utilisé pour vous absenter de vous-même ». Ce double mouvement dans la filature laisse entrevoir une perspective horriblement idéale, où suivi et suiveur s'entretueraient:

Désirait-elle au fond qu'il la tue, que, trouvant cette filature insupportable (surtout parce qu'elle n'escomptait rien, et encore moins une aventure sexuelle), il se jette sur elle, pour lui faire violence, tel Orphée ramenant Eurydice des Enfers, il la fasse soudainement disparaître ${ }^{29}$ ?

C'est le principe même de traque à distance qui suggère cette issue : n'estelle pas motivée par un désir équivoque dès que la poursuite de l'autre s'amorce? Suivre de loin, effleurer l'autre, tout en contrôlant son chemin, résister à l'envie de se faire voir, d'agripper la personne: autant de sentiments qui agitent un parcours insensé et qui dérobe au premier de cordée le même parcours qu’il a tracé seul, devant. L'action consiste, comme dit encore Jean Baudrillard, «à effacer ses traces au fur et à mesure. Or personne ne peut vivre sans traces $3^{\circ}$. » Dans cette grande mise en scène, les tableaux, les souvenirs, les images, l'auteur: tous les éléments assemblés par Sophie Calle participent d'une mise en scène comme suspendue dans le vide, où elle modèle les ombres sur le théâtre photographique, mais toujours à distance. Sur une scène de théâtre, l'auteur est celui qui parle à travers ses personnages, présent à chaque instant mais qui jamais ne se montre: Sophie Calle, plus dramaturge qu'artiste, puise sa matière dans la source de l'absence et sous une ribambelle de déclinaisons, ces vides créent un espace fragmentaire où les représentations de l'identité, de l'œuvre d'art modifient - un peu - l'image de l'artiste, seule persistante car celle des acteurs, quant à elle, reste, à jamais, indéfinie.

29. Jean Baudrillard, «Please follow me», dans Sophie Calle, Suite vénitienne, repris dans Jean Baudrillard, Les stratégies fatales, Paris, Éditions Grasset et Fasquelle, 1983 , p. 145.

30. Jean Baudrillard, Les stratégies fatales, p. 82. 